\title{
Quasi-Elliptic Dual-Band Stepped-Impedance Filters With Folded Parallel High-Impedance Segments
}

\author{
Marjan Mokhtaari\#, Jens Bornemann" ${ }^{\#}$ and Smain Amari* \\ \# Department of Electrical and Computer Engineering, University of Victoria \\ PO Box 3055, Victoria, BC, V8W 3P6, Canada \\ * Department of Electrical and Computer Engineering, Royal Military College of Canada \\ 17000 Station Forces, Kingston, ON, K7K 7B4, Canada
}

\begin{abstract}
A design approach is presented for dual-band filters formed by cascaded stepped-impedance resonators in microstrip technology. The resonators feature folded parallel highimpedance sections which permit resonator coupling in cascaded arrangements and, at the same time, create transmission zeros between the two passbands. Additional transmission zeros in the lower and upper frequency ranges are created by employing source-load coupling. All designs are verified by results obtained from two different commercially available field solvers.
\end{abstract}

\section{INTRODUCTION}

The flexible tuning properties of stepped-impedance resonators (SIR's) are widely known. Therefore, especially in the past few years, dual-band SIR filters found many applications in wireless communication systems. In order to create transmission zeros between and outside of the two passbands, feeding taps at the input/output SIR's of a filter are utilized, e.g. [1], [2]. The same feeding techniques are used in folded cross-coupled designs, [3], [4], in parallel filter topologies [5], and in a number of other arrangements, e.g., [6]-[10]. Except for the cross-coupled filters in [3], [4], the number of achievable transmission zeros between and outside of the passbands is very limited.

The same is true for dual-band SIR filters using parallelcoupled lines at the input/output and between resonators. They either have no transmission zeros, e.g. [11], or a single one past the second passband [12], [13]. Alternatively, a single transmission zero appears between the two passbands [14], [15].

Therefore, this paper focuses on dual-band SIR microstrip filter designs that have at least two transmission zeros between the two passbands and more transmission zeros, added below the first and above the second passband, by utilizing source-load coupling. Coupling takes place by parallel-coupled lines at the input/output. Parallel highimpedance segments are used for coupling between resonators, similar to [16], and some of these segments can be folded for a slightly more compact design.

\section{DESIGN}

Fig. 1a shows the basic stepped-impedance resonator and Fig. $1 b$ its form using folded parallel high-impedance segments. As is well known, the resonant frequencies of a single SIR can be calculated by the following two transcendental equations

$$
R_{z}-\tan \theta_{1} \tan \theta_{2}=0, \quad R_{z} \tan \theta_{1}+\tan \theta_{2}=0
$$

where $R_{z}=Z_{2} / Z_{1}$ is the impedance ratio, and $\theta_{1}$ and $\theta_{2}$ are the electrical lengths of the high and low-impedance segments, respectively. The fundamental and higher order mode resonances occur alternately in the odd and even modes. Their ratio can be determined through (1) in terms of $R_{z}$ and $U$, which is expressed in electrical lengths.

$$
U=\theta_{2} /\left(\theta_{1}+\theta_{2}\right)
$$

Similar to [17], plots are created for the frequencies of higher resonant modes (normalized to the fundamental resonance) versus the length ratio $U$ and for different impedance ratios $R_{z}$. The viable solutions for the length ratios (2) are typically determined with such plots, taking into account - through an appropriate selection for the impedance ratio - that the next higher resonant is positioned sufficiently away from the upper resonance of the dual-band filter. Once $R_{z}$ and $U$ are determined, the high-impedance sections can be realized by parallel lines which might be folded for compactness.

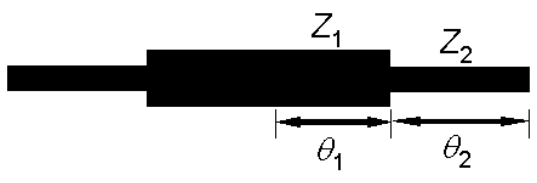

(a)

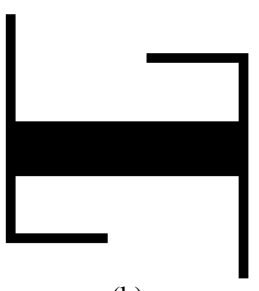

(b)
Fig. 1 Different configurations of a stepped-impedance resonator: basic (a) and using folded parallel high-impedance segments (b).

A passband filter with center frequency $f_{0}$ and fractional bandwidth $\Delta f$ can be characterized by its normalized coupling matrix $M$. De-normalization is typically achieved by

$$
\begin{aligned}
k_{01} & =k_{N, N+1}=M_{01} \sqrt{\Delta f / f_{0}} \\
k_{i j} & =k_{j i}=M_{i j} \Delta f / f_{0}, \quad i, j=1, \ldots, N
\end{aligned}
$$

where $N$ is the number of resonators (nodes) in the filter's coupling topology, and $i=0$ and $j=N+1$ refer to the input and output, respectively.

Since we use parallel-coupled lines as shown in Fig. 2, the coupling element $k_{i j}$ between open-ended coupled-line sections is determined through even- and odd-mode impedances.

$$
k_{i j}=\left(Z_{0 e}-Z_{0 o}\right) /\left(Z_{o e}+Z_{0 o}\right)
$$


In order to design a dual-band passband filter, the coupling matrix is synthesized using the center frequency and bandwidth of the first passband. Due to the electric coupling topology between high-impedance open-ended coupled-line segments [2], it is assumed that the same coupling matrix can be utilized to represent the next passband with the same percentage bandwidth.
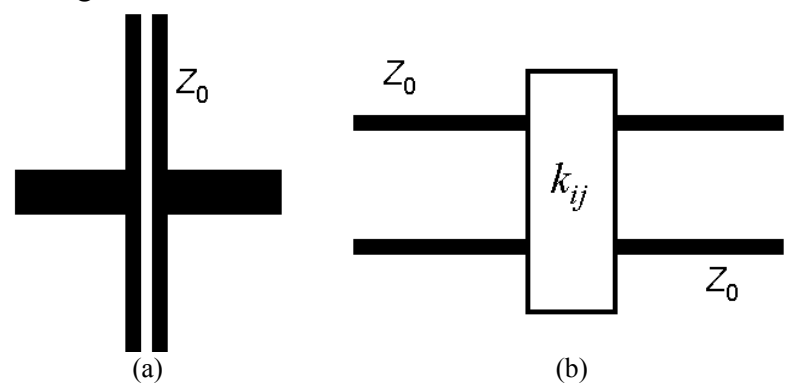

(b)

Fig. 2 Open-ended coupled-line section (a) and its equivalent impedance inverter circuit (b).

Isolation between both passbands is achieved by placing transmissions zeros between them. The coupled-line section in Fig. 2a can generate transmission zeros between the passbands if the length is selected as a quarter wavelength at the arithmetic mean of the two passband frequencies. Note that coupling at the corners of the low-impedance segments in the coupled-line section in Fig. 2a is ignored in the filter synthesis. Therefore, fine-tuning must be considered in the final design.

\section{RESULTS}

The first part of this section describes the basic design approach using coupling elements obtained from standard (e.g. Chebysheff) filter synthesis procedures. Moreover, the creation of transmission zeros between the passbands is presented. The second part introduces additional transmission zeros in the lower and upper frequency ranges.

\section{A. Basic Configurations}

Fig. 3 shows the performance of a dual-band filter using the single SIR configuration indicated in Fig. 1b. The $10 \mathrm{~dB}$ fractional bandwidth is set to $120 \mathrm{MHz}$ for each passband. Two transmission zeros between the two passbands are created by the quarter-wavelength open-ended highimpedance segments. The spurious responses at $6 \mathrm{GHz}$ and $8.5 \mathrm{GHz}$ are due to the second and third higher resonant frequencies of the SIR.

Now consider a dual-band filter design with the same center frequencies but extended $10 \mathrm{~dB}$ fractional bandwidth up to $220 \mathrm{MHz}$ in each passband. Two-cascaded single element SIR's, as depicted in the inset of Fig. 4, are implemented to cover the specifications including sufficient isolation between bands. The normalized coupling elements $M_{01}=M_{23}=1.34$, $M_{12}=2.0$ are obtained for the first passband and are also utilized for the next passband. The gaps between the openended coupled-line sections and input/output paths are determined from the coupling elements and by applying (3) and (4).
Fig. 4 shows the performance of this dual-band filter structure as simulated with two different electromagnetic simulation packages (Ansoft Designer ${ }^{\mathbb{B}}$ and IE3D $^{\circledR}$ ). The computed insertion loss is less than $1.5 \mathrm{~dB}$, and the return loss is better than $12 \mathrm{~dB}$ in each passband. Due to the quarterwavelength open-ended coupled-line sections in the filter configuration (in addition to the open-ended segments of the first and last SIR's), three transmission zeros are generated between the passbands at $2.8 \mathrm{GHz}, 2.9 \mathrm{GHz}$ and $3.125 \mathrm{GHz}$.

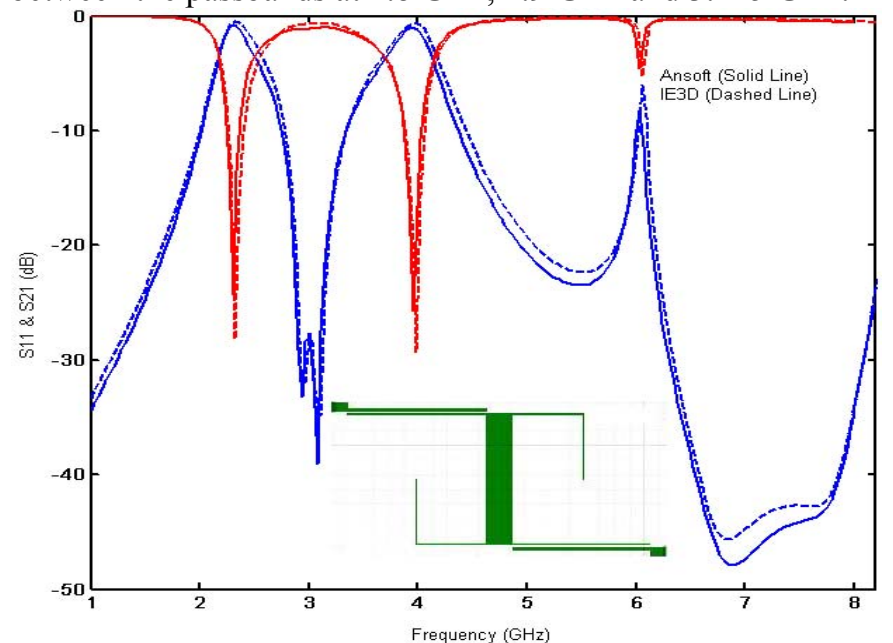

Fig. 3 Performance of a dual-band SIR filter using the single element topology in Fig. 1b.

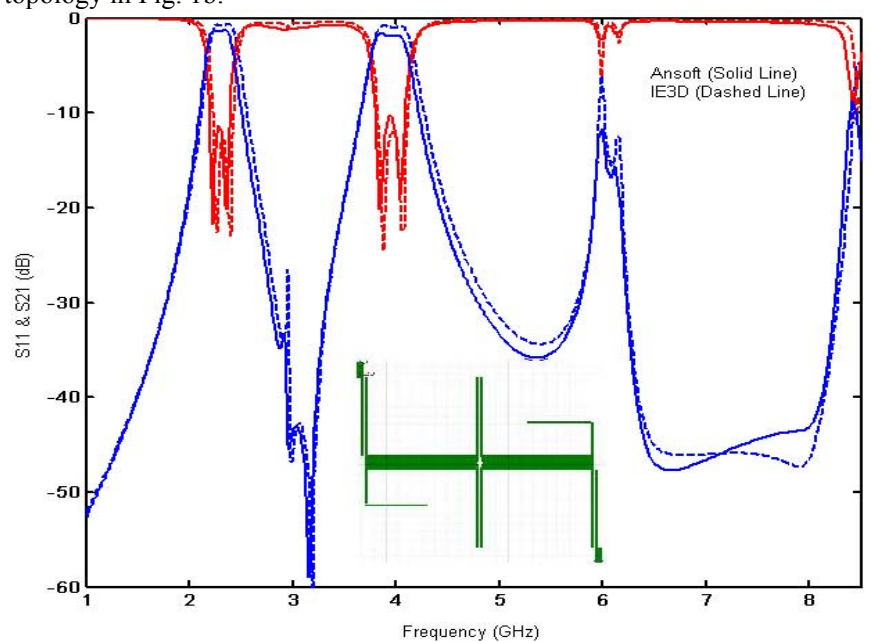

Fig. 4 Performance of a dual-band filter using two cascaded SIR's with folded parallel high-impedance segments.

The design can now be extended to $N$ cascaded SIR's. For instance, for $N=3$ and $10 \mathrm{~dB}$ bandwidths of $300 \mathrm{MHz}$, the normalized coupling elements are $M_{01}=M_{34}=1.035$ and $M_{12}=M_{23}=0.947$. Fig. 5 depicts the corresponding response obtained from Ansoft Designer ${ }^{\circledR}$ and IE3D ${ }^{\circledR}$. The insertion loss in the two passbands is less than $2 \mathrm{~dB}$; the return loss is better than $10 \mathrm{~dB}$. The simulated $10 \mathrm{~dB}$ bandwidths are 300 $\mathrm{MHz}$ and $350 \mathrm{MHz}$. The slightly larger bandwidth in the higher passband is due to the frequency-dependent coupledline sections in the filter structure. This influence is ignored in the dual-band filter synthesis, which assumes the coupling elements to be frequency-independent. Three transmission 
zeros appear between the passbands, resulting in high attenuation and excellent isolation between bands.

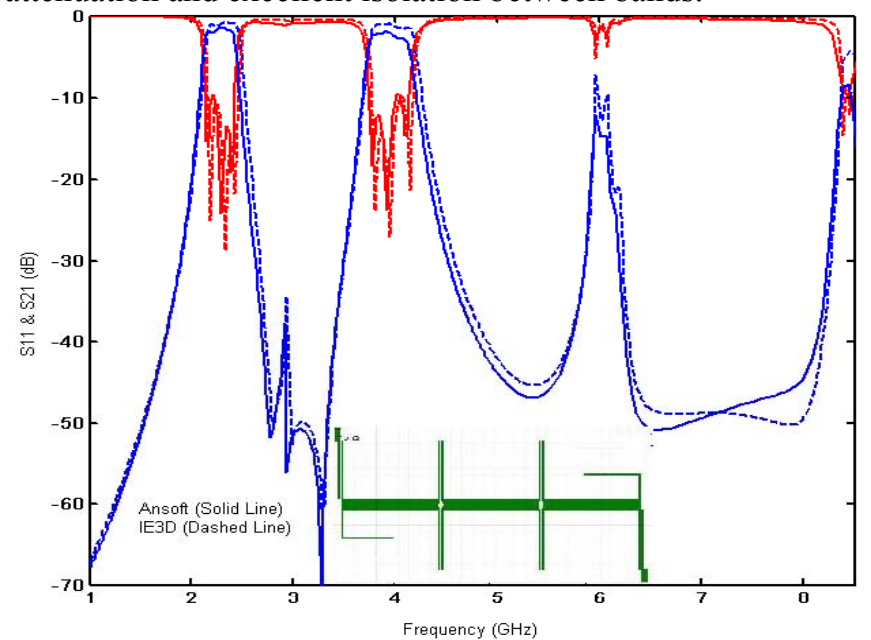

Fig. 5 Performance of a dual-band filter using three cascaded SIR's with folded parallel high-impedance segments.

\section{B. Source-Load Coupling Technique}

Specifications for high performance dual-band filters also call for excellent out-of-band rejection including sharp selectivity of each passband as well as widened/deepened stopbands towards lower and higher frequencies. The previous examples represent dual-band passband filters with multiple transmission zeros between the passbands; however, no transmission zeros are observed in the lower/upper rejection bands. These transmission zeros can be created by introducing source-load coupling. In the following results, which are presented in Fig. 6, we introduce source-load coupling to the designs of Figs. 3, 4 and 5.

Fig. 6a shows the operation of the dual-band filter in Fig. 3 including source-load coupling with the essential dimensions of the dual-band filter in Fig. 3 remaining unchanged. Up to the first spurious response at $6 \mathrm{GHz}$, four transmission zeros (two in the lower rejection band at $1.2 \mathrm{GHz}$ and $2 \mathrm{GHz}$ and two in the upper one at 4.5 and $5.5 \mathrm{GHz}$ ) are created by the magnetic source-load coupling (inset of Fig. 6a). The transmission zeros between the passbands move outwards and improve the selectivity of each passband.

Fig. $6 \mathrm{~b}$ and Fig. $6 \mathrm{c}$ confirm that comparable effects are introduced by using source-load coupling in higher-order SIR filter designs. Compared to Fig. 4 and Fig. 5, two transmission zeros are generated in the lower and two in the upper stopband. The attenuation level of the stopband between the two passbands is reduced. However, since the transmission zeros in this frequency range move outwards, the passbands become more selective.

\section{CONCLUSIONS}

Stepped-impedance resonators with folded parallel highimpedance segments are shown to present attractive solutions for compact dual-band planar filter designs. Cascading individual SIR's via coupled-line sections provides extended bandwidths. In this stage, the design is aided by standard filter

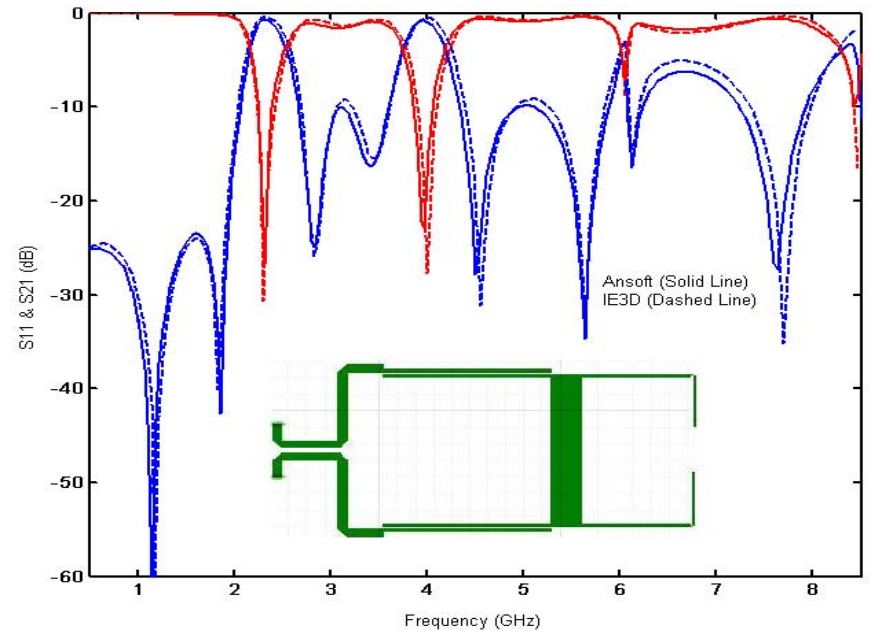

(a)

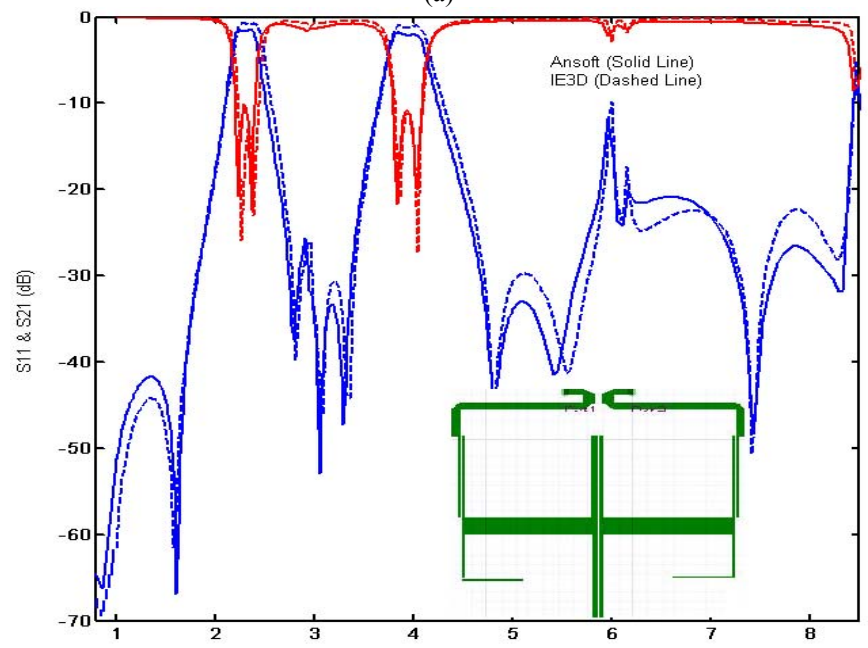

(b)

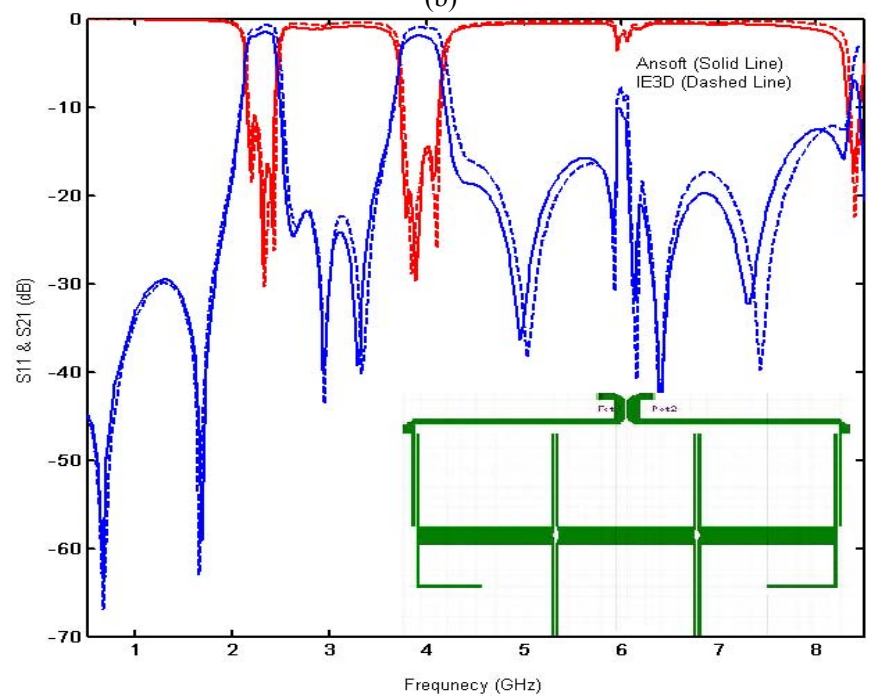

(c)

Fig. 6 Performances of source-load-coupled versions of dual-band filters in Fig. 3 (a), Fig. 4 (b), and Fig. 5 (c).

synthesis. Additionally, multiple transmission zeros typically appear between the two passbands due to the open-ended high-impedance segments and the coupled-line sections. 
Introducing source-load coupling, which not only improves the performance towards lower and higher frequencies, but also enhances the selectivity of each passband, completes the quasi-elliptic design. The single-, dual- and triple-stage SIR dual-band filter designs with and without source-load coupling are verified with two electromagnetic simulation packages. The high performance, compact size and low cost of the new planar dual-band filters demonstrate their suitability and prospective application in dual-band wireless systems.

\section{REFERENCES}

[1] S.-Y. Lee and C.-M. Tsai, "New cross-coupled filter design using improved hairpin resonators", IEEE Trans. Microwave Theory Tech., Vol. 48, pp. 2482-2490, Dec. 2000.

[2] M. Mokhtaari, J. Bornemann and S. Amari, "New reduced-size stepimpedance dual-band filters with enhanced bandwidth and stopband performance", 2006 IEEE MTT-S Int. Microwave Symp. Dig., pp. 11811184, San Francisco, USA, June 2006.

[3] J.-T. Kuo and H.-S. Cheng, "Design of quasi-elliptic function filter with a dual-passband response", IEEE Microwave Wireless Comp. Lett., Vol. 14, pp. 472-474, Oct. 2004.

[4] C.-F. Chen, T.-Y. Huang and R.-B. Wu, "Design of dual- and triplepassband filters using alternately cascaded multiband resonators", IEEE Trans. Microwave Theory Tech., Vol. 54, pp. 3550-3558, Sep. 2006.

[5] C.-Y. Chen, C.-Y. Hsu and H.-R. Chuang, "Design of miniature planar dual-band filter using dual-feeding structures and embedded resonators", IEEE Microwave Wireless Comp. Lett., Vol. 16, pp. 669671, Dec. 2006.

[6] C.-M. Rao, T.-J. Wong and M.-H. Ho, “A parallel doubly coupled dualband bandpass ilter", 2006 IEEE MTT-S Int. Microwave Symp. Dig., pp. 511-514, San Francisco, USA, June 2006.

[7] J.-T. Kuo, T.-H. Yeh and C.-C. Yeh, "Design of microstrip bandpass filters with a dual-passband response", IEEE Trans. Microwave Theory Tech., Vol. 53, pp. 1331-1337, Apr. 2005.

[8] T.-H. Huang, H.-J. Chen, C.-S. Chang, L.-S. Chen, Y.-H. Wang and MP. Houng, "A novel compact ring dual-mode filter with adjustable second-passband for dual-band applications", IEEE Microwave Wireless Comp. Lett., Vol. 16, pp. 360-362, June 2006.

[9] C.-H. Lee, C.-I.G. Hsu and H.-K. Jhuang, "Design of a new tri-band microstrip BPF using combined quarter-wavelength SIRs", IEEE Microwave Wireless Comp. Lett., Vol. 16, pp. 594-596, Nov. 2006.

[10] M.-H. Weng, H.-W. Wu, and Y.-K. Su, "Compact and low loss dualband bandpass filter using pseudo-interdigital stepped impedance resonators for WLANs", IEEE Microwave Wireless Comp. Lett., Vol. 17,2007 , in press.

[11] Y.P. Zhang and M. Sun, "Dual-band microstrip bandpass filter using stepped-impedance resonators with new coupling schemes", IEEE Trans. Microwave Theory Tech., Vol. 54, pp. 3779-3785, Oct. 2006.

[12] S. Sun and L. Zhu, "Coupling dispersion of parallel-coupled microstrip lines for dual-band filters with controllable fractional pass bandwidths", IEEE MTT-S Int. Microwave Symp. Dig., pp. 2195-2198, Long Beach, USA, June 2005

[13] S. Sun and L. Zhu, "Compact dual-band microstrip bandpass filter without external feeds", IEEE Microwave Wireless Comp. Lett., Vol. 15, pp. 644-646, Oct. 2005.

[14] S.-F. Chang, Y.-H. Jeng and J.-L. Chen, "Dual-band step-impedance bandpass filter for multimode wireless LANs", IEE Electron Lett., Vol. 40, pp. 38-39, Jan. 2004.

[15] S. Sun and L. Zhu, "Novel design of dual-band microstrip bandpass filters with good in-between isolation", Proc. Asia-Pacific Microwave Conf., 4 pp., Suzhou, China, Dec. 2005.

[16] Kongpop U-yen, E.J. Wollack, T.A. Doiron, J. Papapolymerou and J. Laskar, "A Planar bandpass filter design with wide stopband using double split-end stepped-impedance resonators", IEEE Trans. Microwave Theory Tech., Vol. 54, pp. 1237-1244, Mar. 2006.

[17] Y.-C. Chiou, J.-T. Kuo and E. Cheng, "Broadband quasi-Chebyshev bandpass filters with multimode stepped-impedance resonators (SIRs)", IEEE Trans. Microwave Theory Tech., Vol. 54, pp. 3352-3358, Aug. 2006. 